\title{
Determinación de Taninos Condensados en Sorgo y su Desactivación Utilizando Urea
}

\author{
NATHALIA OÑA a* FERNANDO NOVILLOb
}

a. Química de Alimentos, Facultad de Ciencias Químicas, Universidad Central del Ecuador, Quito

b. Químico, Facultad de Ciencias Químicas, Universidad Central del Ecuador, Quito

*Correspondencia: nathyru@hotmail.com

\section{Resumen}

El sorgo es una gramínea de origen vegetal que se cultiva en zonas de climas tropicales y subtropicales, y crece en lugares donde los cultivos de maíz no son favorables. Su valor nutritivo es bueno, en comparación con el maíz, en especial por el contenido de proteína. Es por estas razones, que se busca utilizar el sorgo como materia prima para la elaboración de alimentos balanceados para aves, vacunos y cerdos; pero, una limitante es la presencia de taninos condensados en su estructura externa. Estos taninos son polifenoles que forman un complejo con la proteína; así, de esta manera no permite su asimilación y aprovechamiento por parte del animal, para la ganancia de peso. Se puede tratar al sorgo con urea para minimizar este efecto para lo cual se toma en cuenta la influencia de tres variables con dos niveles cada una. Estas variables son el porcentaje de urea al 3 y 6 , el porcentaje de humedad al 13 y 25 , y el tiempo al que se somete el tratamiento 7 y 15 días. Después de haber aplicado cada uno de estos tratamientos, los resultados fueron evaluados de acuerdo al diseño factorial $2^{3}$, y de esta manera se obtuvo que las variables que tienen mayor efecto sobre la disminución del contenido de taninos son urea $6 \%$ y el tiempo de contacto 15 días. A mayor concentración de urea y mayor tiempo de contacto, el contenido de taninos disminuye en un $99,7 \%$.

Palabras Claves: sorgo, taninos, alimentos balanceados, desactivación.

\section{Abstract}

Sorghum grain is a vegetable that is cultivable in tropical and subtropical climate zones, and grows in places where the corn crops are not favorable. Its nutritive value is good, in comparison with the corn, is good, especially because the protein contain. It is for these reasons that we wanted use the sorghum as raw material for the creation of feed for poultry, cattle and pigs, but a limiting factor is the presence of condensed tannins in its exterior structure. These tannins are polyphenols that create a complex with the protein, in that way it does not allow its assimilation and use by animals, for the weight gain. Sorghum can be treated with urea to minimize this effect which is taken in account three variables with two levels each. These variables are the percentage of urea at 3 and 6, the percentage of humidity at 13 and 25, and time that which is referred to the treatment 7 and 15 days. After have applying each on of these treatments, the results were evaluated according to the factorial design $2^{3}$, and in this way was obtained that the variables that have the greatest effect over the reduction of the tannins content is the urea $6 \%$ and the contact time of 15 days. A higher concentration of urea and increased contact time, the tannins content will decline by $99,7 \%$.

Keywords: sorghum, tannins, balanced food, deactivation.

\section{Introducción}

El sorgo es una gramínea similar al maíz, y por lo tanto puede aprovecharse su valor nutricional. Tal es el caso que por el contenido de proteína, el sorgo se puede utilizar en la elaboración de alimentos balanceados para aves, cerdos y rumiantes, siendo una limitante para su uso el porcentaje de taninos condensados, ya que estos forman 
complejos con la proteína y no permiten que el animal la pueda asimilar en su organismo dando como resultado que no haya ganancia de peso.

El uso del sorgo en la elaboración de balanceados no solamente se centra en el beneficio nutricional que este presenta sino también debido a que su costo es menor en relación al del maíz ya que puede ser cultivado en zonas de condiciones no tan favorables para los sembríos de maíz, resistiendo al ataque de plagas debido a los taninos condensados.

Es posible lograr que los taninos condensados presentes en el sorgo se desactiven utilizando urea, ya que el tratamiento físico que recibe durante la molienda no es suficiente para este fin. Este tratamiento químico no solamente disminuiría el contenido de taninos condensados sino que favorece a la preservación de los granos protegiéndolos del ataque por hongos durante el almacenamiento; tomando también en consideración su bajo costo y disponibilidad de este producto.

A nivel mundial, el sorgo granífero (Sorghum bicolor (L) Moench) constituye uno de los cereales que experimenta cada día mayor relevancia agronómica, dadas sus características genotípicas que le permiten ser cultivado incluso bajo condiciones climáticas adversas en las cuales difícilmente podrían desarrollarse otros cultivos de cereales. Tales características han sido asociadas con la presencia de compuestos fenólicos siendo los flavonoides el grupo polifenol más grande presente en este cereal. La producción mundial de sorgo promedio de los años 2002-2003 y 2006-2007 se muestra en el Gráfico 1.

El objetivo de este trabajo es determinar el contenido de taninos condensados utilizando el método colorimétrico Vainilla - Ácido Clorhídrico y desactivar los taninos condensados utilizando urea, para lo cual primero debemos determinar el porcentaje de humedad inicial de las muestras y el porcentaje de taninos condensados, para luego ir evaluando la variación de este contenido en función de la concentración de humedad 13 y $25 \%$, adición de urea 3 y $6 \%$, y el tiempo de tratamiento 7 y 15 días.

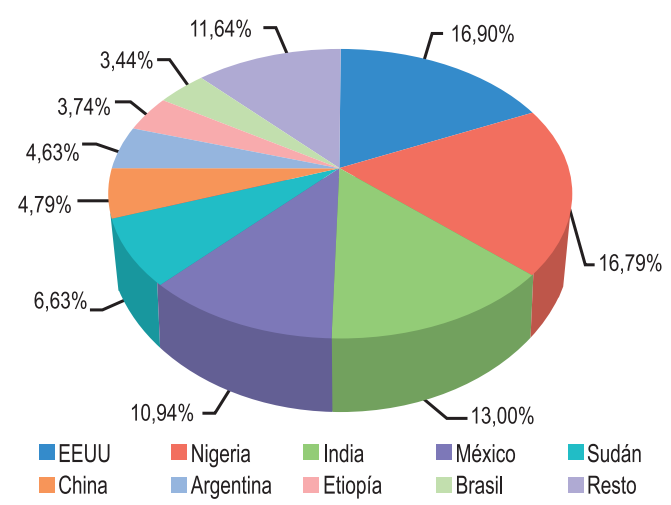

Gráfico 1. Producción Mundial de Sorgo
(2002/03-2006/07)
Fuente: www.sagpya.mecon.gov.ar

Un sorgo con alto contenido de taninos condensados tendrá la capacidad de precipitar o fijar más proteína de la existente en los mismos; siendo así que aquellos sorgos sin taninos o con un porcentaje bajo tienen un valor nutritivo equivalente al del valor nutritivo del maíz, pudiendo sustituirlo completamente en la formulación nutricional.

En la formulación de balanceados el sorgo se ubica como un cereal de preferencia en la alimentación animal de pollos, cerdos y rumiantes debido a su disponibilidad; además, se incrementa su valor nutritivo cuando está debidamente procesado.

Busca aplicarse el método químico de desactivación de taninos condensados con urea, con el propósito de conseguir los beneficios nombrados anteriormente sobre todo en relación a que hay menor gasto de recursos en comparación a una desactivación con vapor donde se consume más energía.

\subsection{Sorgo}

El sorgo o zahína (Sorghum bicolor) es una hierba perteneciente a la familia de las gramíneas (Poaceae), cuyas semillas se utilizan para hacer harina y como forraje. Su clasificación científica la podemos ver en la Tabla 1.

El género Sorghum bicolor se caracteriza por presentar espiguillas que nacen de a pares. El sorgo tiene una 
altura de 1 a 2 metros. Tiene inflorescencias en panojas y semillas de $3 \mathrm{~mm}$, esféricas y oblongas, de color negro, rojizo y amarillento. Tiene un sistema radicular que puede llegar en terrenos permeables a $2 \mathrm{~m}$ de profundidad. Las flores tienen estambres y pistilos; pero, se han encontrado en Sudán sorgos dioicos. El sorgo se utiliza para producir grano que sirve para la alimentación del ganado [1].

Tabla 1. Clasificación Científica del Sorgo [1].

\begin{tabular}{ll}
\hline \multicolumn{2}{c}{ CLASIFICACIÓN CIENTÍFICA } \\
\hline Reino & Plantae \\
División & Magnoliophyta \\
Clase & Liliopsida \\
Orden & Poales \\
Familia & Poaceae \\
Subfamilia & Panicoideae \\
Tribu & Andropogonea \\
Género & Sorghum \\
& Moencha \\
Especie & Sorghum bicolor \\
\hline
\end{tabular}

Alrededor del $90 \%$ de almidón y $80 \%$ de proteína total están localizados en el endospermo; el germen contiene a su vez el $70 \%$ de extracto etéreo. En conjunto el sorgo posee una composición similar a la del maíz, excepto por un menor contenido de aceite. La composición química aproximada del grano de sorgo se indica en la Tabla 2.

Tabla 2. Composición Química del Grano de Sorgo [2].

\begin{tabular}{lc}
\hline \multicolumn{2}{c}{ PARÁMETRO } \\
QUÍMICO & RAN) \\
\hline PC (Nx6,25) & $7,01-12,54$ \\
Almidón & $57,44-76,31$ \\
Cenizas & $1,13-4,78$ \\
Calcio & $0,03-0,11$ \\
Fósforo & $0,25-0,71$ \\
Taninos & $0,55-2,70$ \\
condensados (EC) & $2,64-6,40$ \\
FC & $11,72-37,19$ \\
FDN & $6,66-18,48$ \\
FDA & $2,93-8,93$ \\
Lignina & $3,45-9,26$ \\
Celulosa &
\end{tabular}

El sorgo tiene más proteína y menos aceite que el maíz, lo cual se traduciría en un contenido de energía metabolizable ligeramente inferior. Los sorgos graníferos sin taninos condensados tienen un valor nutritivo equivalente al $95-98 \%$ del valor nutritivo del maíz [2]. Para obtener el máximo de eficiencia alimenticia, los granos del sorgo deberán ser procesados (silaje de grano húmedo, molido, aplastado, etc.).

En los sorgos de alto tenor de taninos, la testa (porción externa del tegumento exterior) se presenta prominente y coloreada, violácea a marrón rojiza, por la presencia de estas sustancias, constituyendo un rasgo diferencial respecto de aquellos que no presentan taninos (testa incolora) [3].

Valores de referencia para taninos condensados en sorgo se presentan en la Tabla 3.

Tabla 3. Contenido de Taninos en Sorgo [3].

\begin{tabular}{c|c}
\hline $\begin{array}{c}\text { CONTENIDO DE } \\
\text { TANINOS }\end{array}$ & $\begin{array}{c}\text { EQUIVALENTE } \\
\text { CATEQUINA (\%) }\end{array}$ \\
\hline BAJO & Menos 0,95 \\
MEDIO & 0,95 a 1,90 \\
ALTO & Más de 1,90 \\
\hline
\end{tabular}

\subsection{Taninos}

De la palabra inglesa tanning (curtido), el término tanino fue originalmente usado para describir la sustancia de los extractos vegetales usados para curtir cueros animales.

Se define a los taninos como compuestos fenólicos de alto peso molecular y con una cantidad adecuada de grupos hidroxi-fenólicos y otros grupos sustituibles como grupos carboxilos, para formar complejos resistentes con proteínas y otras macromoléculas. La palabra fenol es referida a todos aquellos compuestos que poseen uno o más radicales hidroxilo, sustituyentes unidos a un anillo aromático [4].

La principal característica de los taninos es que pueden ligarse a las proteínas. Históricamente se creía que los taninos ligaban y precipitaban todas las proteínas de 
manera no específica; pero, ahora se reconoció que las interacciones tanino-proteína son específicas y dependen de la estructura de la proteína. Una proteína de cadena más larga es favorable para la formación del complejo; esta interacción se intensifica con la movilidad conformacional y el peso molecular de los taninos [5].

La incidencia de los taninos en aves, cerdos y rumiantes puede tener efectos benéficos y periudiciales. Entre estos últimos se destacan:

- Inhibición microbiana y enzimática debido a un exceso de ácido tánico en la dieta.

- Colores indeseables en el alimento.

- Insolubilización y precipitación de las proteínas.

- Depresión de la digestibilidad de la materia seca y la utilización del nitrógeno.

- Depresión de la energía.

- Provocan carcinogénesis.

- Disminución de la palatabilidad [6].

Pero, estas sustancias presentan algunas aplicaciones tanto en medicina cuanto en la industria. A continuación se nombra algunas de estas ventajas:

- Curación de heridas y cuidados de la piel. Los taninos cumplen una función cicatrizante al acelerar la curación de las heridas y hemostática al detener el sangrado.

- Antioxidantes. Se los considera así por su capacidad para eliminar los radicales libres, previniendo la aparición de numerosas enfermedades degenerativas, entre ellas el cáncer.

- Antibacterianas. Esta función se da al privar a los microorganismos del medio apropiado para que puedan desarrollarse.

- Colesterol. Los taninos reducen el colesterol al inhibir su absorción y expulsarlos a través de las heces.

- En la industria vinífera los taninos son de gran importancia, sirven como conservantes, es decir, que determinan la capacidad de envejecimiento de un vino [7].

\subsection{Determinación de Taninos Condensados}

Existen algunos métodos para determinar compuestos fenólicos como son los taninos condensados. Entre estos pueden tomarse en cuenta los colorimétricos que son usados para medir sorgos con taninos, estos incluyen la prueba de Vainillina/HCl [8] y la prueba de $\mathrm{HCl} / \mathrm{Bu}$ tanol, ambos son rápidos y económicos para estimar el contenido de taninos.

El método vainillina-HCl es el más utilizado para la determinación cuantitativa de los taninos condensados en frutas, sorgo y leguminosas forrajeras. Este análisis es específico para determinar flavan-3-ol, dihidrochalconas y proantocianidinas [9].

\subsection{Desactivación de Taninos Condensados}

La desactivación de los taninos condensados consiste en disminuir su contenido en el grano de sorgo; sea cual fuera el método aplicado siempre el fin es buscar un mejor rendimiento, bajos costos y que el efecto que tiene sobre el valor nutricional del grano no se vea afectado y así poder aprovechar la proteína que este presenta.

Muchos esfuerzos han sido dirigidos a tratar de mejorar la calidad nutritiva del grano de sorgo, para lo cual algunos estudios han evaluado distintas técnicas de tratamiento basadas en la utilización de métodos físicos y/o químicos dirigidos a disminuir el contenido de taninos [10]. Entre los métodos químicos, se destaca el que utiliza la urea, consiste en agregar la urea en una proporción del tres por ciento de la cantidad de grano, y la cantidad necesaria de agua para reconstruir el grano a un nivel entre el veinticinco por ciento de humedad. Debe cuidarse que la urea no contenga nitratos o nitritos, ya que son tóxicos para los animales [11].

Los taninos condensados pueden ser desactivados rápida y completamente por la reconstitución con soluciones acuosas de urea, siendo también un buen preservante para las muestras. La temperatura tiene efecto sobre la desactivación de los taninos con urea según se demuestra en un experimento realizado, donde se utilizó soluciones de dos, tres y cuatro por ciento de urea que se mantuvieron a $25^{\circ} \mathrm{C}$; hubo disminución en el porcentaje de taninos; pero, no mayor diferencia entre una concentración y la otra. Luego se varió la temperatura, se subió a $60^{\circ} \mathrm{C}$ a todas las muestras obteniéndose una mayor disminución en los valores de taninos a esta temperatura [12]. 


\section{Materiales y Métodos}

\subsection{Determinación de Taninos Condensados}

\subsubsection{Materiales y Equipos}

- Espectrofotómetro (Milton Roy Company)

- Baño térmico con control de temperatura 100+/$0,5^{\circ} \mathrm{C}$ (MEMMERT)

- Agitador magnético

- Centrífuga (DYNAC)

\subsubsection{Reactivos}

- Solventes: Metanol grado reactivo (Merck), ácido clorhídrico concentrado comercial (Merck)

- Estándar primario al 98\% de Hidrato de Catequina grado vainillina (Sigma Chemical).

- Solución de vainillina al 1\% p/v.- Se pesa 1,0 gramos de vainillina y se llevan a $100 \mathrm{~mL}$ con metanol. Se guarda la solución en un recipiente oscuro a $0^{\circ} \mathrm{C}$

- Solución de ácido clorhídrico al 8\% v/v.- Se toman $8 \mathrm{~mL}$ de ácido clorhídrico concentrado y se completa con metanol hasta $100 \mathrm{~mL}$.

- Solución de ácido clorhídrico al $4 \%$ v/v.- Se toman $4 \mathrm{~mL}$ de ácido clorhídrico concentrado y se completa con metanol hasta $100 \mathrm{~mL}$.

- Solución patrón de catequina.- se prepara una solución cuya concentración sea de $3 \mathrm{mg} / \mathrm{mL}$ de catequina en metanol.

- Reactivo de vainillina.- Debe prepararse el mismo día del análisis mezclando partes iguales de la solución de vainillina y $\mathrm{HCl}$ al $8 \%$.

\subsubsection{Procedimiento}

- Se toma una alícuota de $1 \mathrm{~mL}$ de extracto (por duplicado) en tubos de ensayo y se colocan en un baño de agua a $30^{\circ} \mathrm{C}$

- Después de que las muestras se han atemperado se agregan $5 \mathrm{~mL}$ de reactivo de vainillina a uno de los tubos y $5 \mathrm{~mL} \mathrm{de} \mathrm{HCl} 4 \%$ a otro tubo (blanco) a intervalos de 1 minuto entre una muestra y la siguiente.

- Las muestras con sus respectivos blancos se dejan en un baño de agua a $30^{\circ} \mathrm{C}$ por espacio de 20 minutos exactos.
- La absorbancia del blanco corresponde a la absorbancia de la muestra que contiene la vainillina

- La diferencia en absorbancia se compara con la obtenida en la curva patrón para hallar el equivalente en catequina.

- Si la absorbancia de la muestra no cae dentro del intervalo de absorbancia de la curva, se diluye la muestra de tal forma que su nuevo valor de absorbancia pueda interpretarse en la curva de la calibración.

- Para la cuantificación se interpreta la absorbancia de la muestra en la curva patrón y se obtienen los mg de catequina equivalente y los mililitros de extracto. Ecuación (1).

$$
\% \text { Taninos }=\frac{(\mathrm{mgCatequinax} 10 \mathrm{mLx} 100)}{(1 \mathrm{mLxPM}(\mathrm{mg}))}
$$

\subsection{Desactivación de Taninos Condensados}

\subsubsection{Reactivos}

Urea grado técnico

\subsubsection{Procedimiento}

- Determinar el porcentaje de humedad de las muestras de sorgo según la Norma INEN 518 [13].

- Determinar el porcentaje de taninos de acuerdo con la técnica descrita anteriormente.

- Realizar los cálculos de balance de materia y humedecer las muestras con las soluciones acuosas de urea al 3 y $6 \%$ para obtener el 13 y $25 \%$ de humedad.

- Conservar las muestras durante 7 y 15 días a temperatura ambiente en los frascos de plástico bien tapados, para evitar la pérdida o ganancia de humedad.

- Determinar el pH de la muestra agregada urea.

- Analizar el porcentaje de taninos después del tiempo indicado para cada tratamiento según la técnica descrita anteriormente.

El resumen de los tratamientos realizados se indica en la Tabla 4. 


\begin{tabular}{ccccc} 
Tabla 4. Combinaciones para cada tratamiento. \\
I KAI. & $\begin{array}{c}\text { I Iciviru } \\
\text { (días) }\end{array}$ & $\begin{array}{c}\text { UKcA } \\
\text { (\%) }\end{array}$ & $\begin{array}{c}\text { nuvicuAu } \\
\text { (\%) }\end{array}$ & $\begin{array}{c}\text { \# } \\
\text { REP. }\end{array}$ \\
\hline 1 & 7 & 3 & 13 & 4 \\
2 & 15 & 3 & 13 & 4 \\
3 & 7 & 3 & 25 & 4 \\
4 & 15 & 3 & 25 & 4 \\
5 & 7 & 6 & 13 & 4 \\
6 & 15 & 6 & 13 & 4 \\
7 & 7 & 6 & 25 & 4 \\
8 & 15 & 6 & 25 & 4
\end{tabular}

\subsubsection{Balance de Materia}

Para poder llegar a las condiciones adecuadas y llevar a cabo la desactivación de los taninos condensados es necesario realizar un balance de materia para conocer en qué cantidades va a intervenir cada elemento.

\section{Cálculos}

$$
\begin{aligned}
& A=\text { sorgo }(\mathrm{g}) \\
& B=\text { urea }(\% \mathrm{P} / \mathrm{V}) \\
& C=\operatorname{agua}(\mathrm{g}) \\
& D=\text { producto final }(\% \text { de humedad })
\end{aligned}
$$

Ejemplo de cálculo:

- $3 \%$ de urea y $13 \%$ de humedad del producto final

$$
A+B+C \rightarrow D
$$

$$
\begin{aligned}
& 3+0,09+C=D \\
& 3,09+C=D(1) \\
& \text { Balance de masa } \mathrm{H}_{2} \mathrm{O} \\
& (0.11)(3)+0+\mathrm{C}=0,13 \mathrm{D}
\end{aligned}
$$

$$
X_{\mathrm{H}_{2} \mathrm{OA}}{ }^{\mathrm{A}}+\mathrm{X}_{\mathrm{H}_{2} \mathrm{OB}}{ }^{\mathrm{B}+} \mathrm{H}_{\mathrm{H}_{2} \mathrm{OC}} \mathrm{C}=X_{\mathrm{H}_{2} \mathrm{OD}}{ }^{D}
$$

$$
\begin{aligned}
& 0,33+C=0,13 D \\
& (1) \text { y }(2) \\
& -3,09-C=-D \quad(1) \\
& 0,33+C=0,13 D(2) \\
& -2,76=-0,87 D
\end{aligned}
$$

$\mathrm{D}=3,17 \mathrm{~g}$ total

$A=3 g$

$\mathrm{B}=0,09 \mathrm{~g}$

$\mathrm{C}=0,08 \mathrm{~g}$

Los cálculos se realizan de acuerdo al porcentaje de urea y de humedad con las que van a trabajarse.

\section{Resultados y Discusión}

\subsection{Determinación de Humedad}

En la Tabla 5 se indica el porcentaje de humedad para cada una de las muestras.

Tabla 5. \% Humedad Muestras.

\begin{tabular}{c|c|c}
\hline Valor & Muestra $\mathbf{a}$ & Muestra b \\
\hline$\%$ Humedad & 11,25 & 11,33
\end{tabular}

El valor medio de humedad de las muestras es de $11,29 \%$, de acuerdo a valores de referencia de la Norma del CODEX para el sorgo en grano CODEX STAN 172-1989 es de 14,5\% como valor máximo, lo que indica que cumple con la misma.

\subsubsection{Determinación de Taninos Condensados.}

En la Tabla 6 se indica el porcentaje de taninos condensados inicial para cada una de las muestras.

Tabla 6. \% Taninos Condensados Muestras a y $b$

\begin{tabular}{ccc}
\hline Valor & Muestra $\boldsymbol{a}$ & Muestra $\boldsymbol{b}$ \\
\hline$\%$ Taninos & 1,92 & 2,47 \\
\hline
\end{tabular}

El contenido promedio de taninos condensados de las muestras es de 2,20\%, y de acuerdo al Manual Técnico del sorgo que lo clasifica según se indica en la Tabla 3, las muestras tienen un contenido alto de taninos.

El diseño estadístico utilizado es el Diseño Factorial, donde intervienen 3 variables con dos niveles cada una utilizándose el modelo $2^{3}$, con lo que se determina que va a tenerse ocho tratamientos donde se combinan cada una de las variables. 
Se trabaja con dos muestras $a$ y $b$, con cuatro réplicas para cada uno de los tratamientos que se aplican, los mismos que resultan de la combinación del porcentaje de humedad ( 13 y 25), porcentaje de urea ( 3 y 6 ) y el tiempo en días al que se somete cada tratamiento $(7 y$ 15). Las lecturas se realizan en el espectrofotómetro a $500 \mathrm{~nm}$, se obtienen los valores de absorbancia los mismos que se transforman a mg de catequina con ayuda de la ecuación que se obtiene en la curva de calibración de acuerdo con la Norma Venezolana COVENIN3179:1995 [8]. De acuerdo con esto se hace el cálculo del contenido de taninos inicial y final.

Tabla 7. \%Taninos para cada tratamiento Muestra a.

\begin{tabular}{ccccccc}
\hline \multicolumn{7}{c}{ Muestra $\mathbf{a}$} \\
\hline$\#$ & $\mathbf{X}_{\mathbf{1}}$ & $\mathbf{X}_{\mathbf{2}}$ & $\mathbf{X}_{\mathbf{3}}$ & $\mathbf{X}_{\mathbf{4}}$ & SUMA & MEDIA \\
\hline 1 & 0,490 & 0,400 & 0,190 & 0,210 & 1,290 & 0,3225 \\
2 & 0,010 & 0,020 & 0,050 & 0,100 & 0,180 & 0,0450 \\
3 & 0,180 & 0,030 & 0,060 & 0,030 & 0,300 & 0,0750 \\
4 & 0,010 & 0,050 & 0,020 & 0,020 & 0,100 & 0,0250 \\
5 & 0,550 & 0,490 & 0,190 & 0,290 & 1,520 & 0,3800 \\
6 & 0,020 & 0,010 & 0,010 & 0,040 & 0,080 & 0,0200 \\
7 & 0,020 & 0,140 & 0,050 & 0,030 & 0,240 & 0,0600 \\
8 & 0,010 & 0,060 & 0,024 & 0,011 & 0,105 & 0,0263 \\
\hline \multicolumn{7}{c}{ Suma } \\
\hline
\end{tabular}

$\mathrm{X}_{1,2,3,4}=$ número de réplicas

I = suma del resultado de todos los tratamientos

Tabla 8. \% Taninos para cada tratamiento Muestra b.

\begin{tabular}{ccccccc}
\hline \multicolumn{7}{c}{ Muestra $\boldsymbol{b}$} \\
\hline$\#$ & $\mathbf{X}_{1}$ & $\mathbf{X 2}$ & $\mathbf{X 3}$ & $\mathbf{X} 4$ & SUMA & MEDIA \\
\hline 1 & 0,710 & 0,400 & 0,150 & 0,240 & 1,500 & 0,3750 \\
2 & 0,010 & 0,020 & 0,020 & 0,050 & 0,100 & 0,0250 \\
3 & 0,210 & 0,165 & 0,140 & 0,050 & 0,565 & 0,1413 \\
4 & 0,070 & 0,060 & 0,040 & 0,040 & 0,210 & 0,0525 \\
5 & 0,150 & 0,160 & 0,230 & 0,260 & 0,800 & 0,2000 \\
6 & 0,035 & 0,034 & 0,020 & 0,040 & 0,129 & 0,0323 \\
7 & 0,065 & 0,100 & 0,050 & 0,100 & 0,315 & 0,0788 \\
8 & 0,010 & 0,029 & 0,030 & 0,0001 & 0,069 & 0,0173 \\
\hline \multicolumn{5}{c}{ Suma } & $I=$ & 3,688 \\
\hline
\end{tabular}

$\mathrm{X}_{1,2,3,4}=$ número de réplicas

$I$ = suma del resultado de todos los tratamientos
En las Tablas 7 y 8 tenemos el porcentaje de taninos condensados después de aplicar cada uno de los tratamientos para la Muestra a y la Muestra b respectivamente.

En los Gráficos 2 y 3 podemos observar la disminución del porcentaje de Taninos en función de la concentración de urea (3 y $6 \%)$, el porcentaje de humedad (25\%) y el tiempo de contacto (15 días).

En los gráficos para cada uno de los tratamientos se ve la tendencia que tiene el contenido de taninos condensados en ir disminuyendo a medida que aumenta la cantidad de urea añadida, teniendo también influencia el porcentaje de humedad y el tiempo de contacto de cada tratamiento.

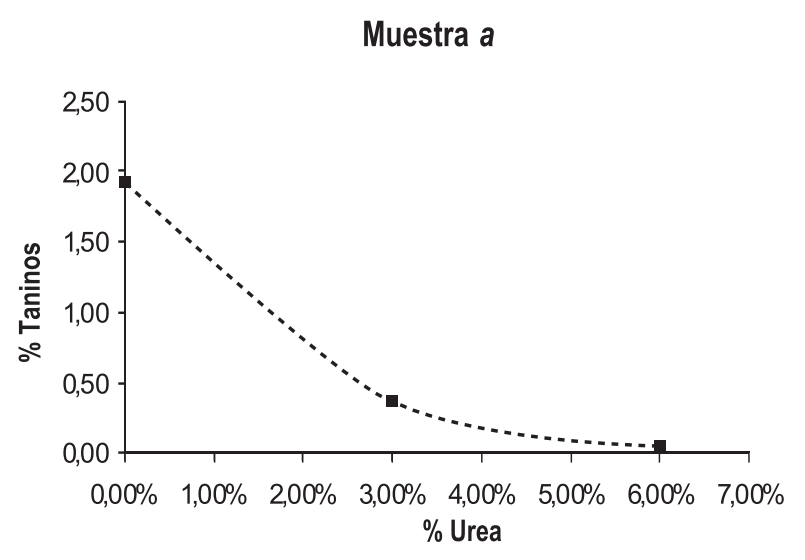

Gráfico 2. Disminución de taninos a 3 y $6 \%$ de urea a 15 días $25 \%$ humedad.

\section{Muestra $b$}

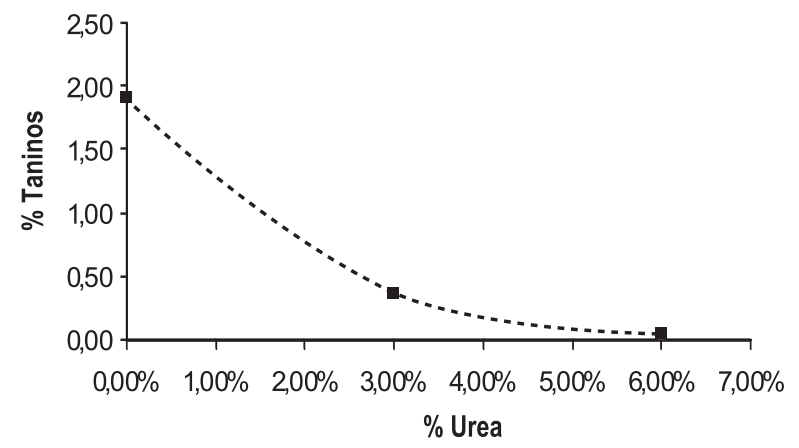

Gráfico 3. Disminución de taninos a 3 y $6 \%$ de urea a 15 días $25 \%$ humedad. 


\subsubsection{Análisis de Varianza}

El tratamiento estadístico se realiza de acuerdo a las fórmulas indicadas para el diseño factorial $2^{3}$.

Para la aceptación se utiliza la Prueba F al 95\%, F para 1 y 24 grados de libertad el valor en tablas es de 4,26. Los tratamientos son:

$\mathrm{A}=\%$ humedad

$\mathrm{B}=\%$ urea

$\mathrm{C}=$ días

Los demás resultan de la combinación de estos tres.

Tabla 9. F calculada para cada tratamiento Muestra a.

\begin{tabular}{|c|c|c|}
\hline TRATAMIENTOS & $F$ calculada & $F$ tabulada \\
\hline A & 0,02 & 4,26 \\
\hline B & $20,10^{*}$ & 4,26 \\
\hline C & $30,95^{*}$ & 4,26 \\
\hline$A B$ & 0,13 & 4,26 \\
\hline$A C$ & 0,26 & 4,26 \\
\hline$B C$ & $18,24^{*}$ & 4,26 \\
\hline$A B C$ & 0,58 & 4,26 \\
\hline
\end{tabular}

* Tratamientos aceptados

Tabla 10. F calculada para cada tratamiento Muestra $\mathbf{b}$.

\begin{tabular}{ccc}
\hline TRATAMIENTOS & $\boldsymbol{F}$ calculada & $\boldsymbol{F}$ tabulada \\
\hline A & 3,71 & 4,26 \\
B & $\mathbf{6 , 1 8 ^ { * }}$ & 4,26 \\
C & $\mathbf{2 3 , 5 1 *}^{*}$ & 4,26 \\
AB & 0,26 & 4,26 \\
AC & 0,26 & 4,26 \\
BC & $\mathbf{7 , 1 2 ^ { * }}$ & 4,26 \\
ABC & 1,27 & 4,26 \\
\hline
\end{tabular}

* Tratamientos aceptados

En las Tablas 9 y 10 puede verse que de acuerdo al valor de $\mathrm{F}$ calculado en comparación al $\mathrm{F}$ tabulado al $5 \%$, se aceptan los tratamientos B (\%urea), C (días) y la combinación de ambos $B C$, lo que indica que estas son las variables que tienen mayor influencia sobre la disminución de taninos.

Después de haber concluido cuales son las variables que influyen sobre la disminución del porcentaje de taninos, se crea una gráfica de superficie de respuesta (Gráfico 2 y 3), la que nos indica que a medida que el porcentaje de urea va aumentando y los días también nos vamos acercando más al cero por ciento de taninos; esto se observa por los cambios de color que se ve en la superficie. El color rojo corresponde a mayor concentración de taninos y el color verde a una menor concentración.

Los gráficos de superficie de respuesta se realizaron con el programa STATISTICA 7.

La ecuación que se obtiene con el gráfico de superficie de respuesta permite calcular el porcentaje de taninos que se obtienen al variar la concentración de urea $(x)$ y los días (y), es decir, que permite conocer con qué valor de urea y en qué tiempo puede obtenerse una total disminución del contenido de taninos.

$x=\%$ urea
$y=$ días

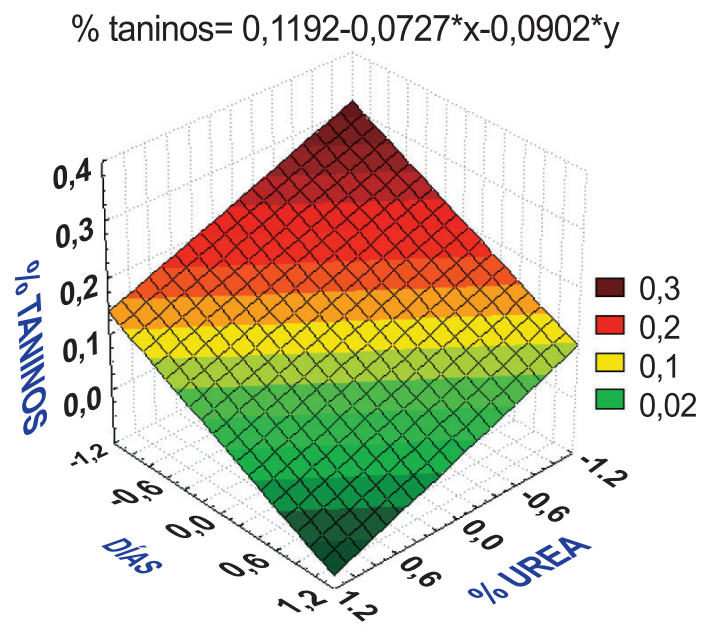

Gráfico 2. 3D Muestra a.

$$
\% \text { taninos }=0,1117-0,045^{*} x-0,0798^{*} y
$$

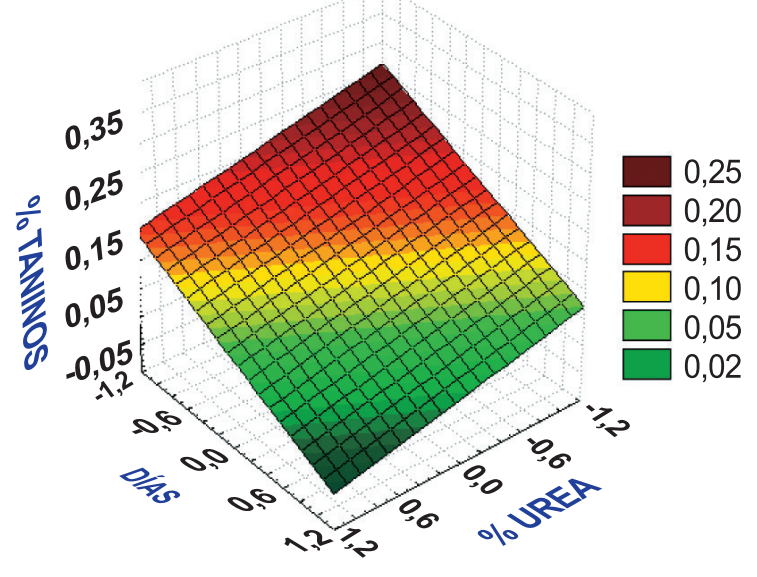

Gráfico 3. 3D Muestra b. 
Para poder confirmar si el porcentaje de taninos va disminuyendo a medida que aumenta el porcentaje de urea, se realizó pruebas experimentales fuera de lo propuesto en el trabajo, donde se trabajó con concentraciones de 1,5 y $9 \%$ de urea en las mismas condiciones en las que se trabajó los demás tratamientos. De acuerdo a la tendencia de disminuir el contenido de taninos en función del tiempo se realizó una determinación a los 10 días de tratamiento bajo las mismas condiciones. Para conocer si la temperatura tiene influencia sobre la disminución de taninos condensados se realizó las determinaciones a $35^{\circ} \mathrm{C}$. los resultados los podemos observar en la Tabla 11 .

Tabla 11. Influencia una variable a la vez.

\begin{tabular}{cccc}
\hline \multicolumn{2}{c}{ Variable $/$ Muestra } & $\begin{array}{c}\text { \% T.C } \\
\text { (a) }\end{array}$ & $\begin{array}{c}\text { \% T.C } \\
\text { (b) }\end{array}$ \\
\hline \multirow{2}{*}{$\%$ Urea } & 1,5 & 0,88 & 0,82 \\
& 9 & 0,12 & 0,14 \\
\hline Tiempo & & & 0,14 \\
$\mathrm{~T}\left({ }^{\circ} \mathrm{C}\right)$ & 35 & 0,01 & 0,001 \\
\hline
\end{tabular}

De acuerdo con la influencia que tiene la urea sobre el contenido de taninos, se decidió aumentar dos valores de urea 1,5 y $9 \%$, y determinar si dicha tendencia se mantiene. Con el nivel inferior utilizado se observa una disminución del $60,45 \%$ valor promedio de ambas muestras, y con el nivel superior tenemos una disminución del 94,06\% valor promedio de ambas muestras. Con los resultados que se obtienen, se confirma el efecto de la urea sobre la disminución del contenido de taninos.

Otra de las variables que afecta al contenido de taninos es el tiempo de contacto al que se somete las muestras, se incluyó un tiempo medio de 10 días, que va dentro de los valores utilizados para confirmar la disminución de taninos en función del tiempo. De acuerdo con los valores que se obtienen a los 10 días puede determinarse que hay una relación directa en el tiempo de contacto y la disminución de taninos.

Para conocer sí la temperatura tiene influencia sobre la concentración de taninos se probó a $35^{\circ} \mathrm{C}$ a las condiciones establecidas. La temperatura reduce en un $99,9 \%$ el porcentaje de taninos. Confirmando así lo que indicaron Russel y Lolley en su artículo [14], determinaron que la temperatura tiene efecto sobre la disminución de taninos condensados.

\section{Conclusiones}

- El porcentaje de humedad que presenta cada muestra es de $11,25 \%$ para la muestra a y $11,37 \%$ para la muestra $b$. Este dato sirvió de referencia para saber cuánto de agua debía aumentarse para poder llegar a las humedades 13 y $25 \%$, requeridas para cada uno de los tratamientos.

- La determinación de taninos condensados en el sorgo procedente de Quevedo, de la zona de Balzar, realizada por el método colorimétrico $\mathrm{HCl}$-vainillina dio como resultado para la muestra a 1,92\% TC; y de la muestra b 2,47\% TC.

- La desactivación de los taninos condensados se dio con cada una de las posibles combinaciones de las variables de humedad al 13 y $25 \%$; soluciones acuosas de urea al 3 y $6 \%$; y el tiempo de tratamiento 7 y 15 días. Obteniéndose como mejor resultado que a mayor concentración de urea y mayor tiempo de contacto el valor de taninos disminuye, la Muestra a disminuye en un $99,4 \%$ y la Muestra $b$ disminuye en un $99,9 \%$.

- La urea por el tiempo que está en contacto con el sorgo produce un álcali debido a su hidrólisis, la misma que se favorece por la presencia del agua añadida. El pH del medio se eleva a un valor de 8,3 aproximadamente lo que impide el desarrollo de hongos, que son los principales microorganismos que atacan al grano.

- Del análisis estadístico de todos los posibles tratamientos se concluye que la concentración de urea y el tiempo de contacto y también una combinación de ambos, tienen mayor influencia sobre la desactivación de los taninos. Esto pudo ser determinado mediante la prueba $\mathrm{F}$ al $5 \%$ en donde $\mathrm{F}$ tabulado para 1 y 24 grados de libertad es de 4,26. Se acepta la hipótesis nula para la humedad lo que indica que la variación de esta no tiene mayor efecto sobre el contenido de taninos con los niveles investigados en este trabajo. Se acepta la hipótesis alternativa para el porcentaje de urea y su combinación, que indica que un valor diferente a cero disminuye el contenido de taninos.

- De acuerdo con los gráficos 2 y 3, porcentaje de urea vs. porcentaje de taninos, puede observarse una disminución progresiva de taninos a medida que aumenta la concentración de urea, y la influencia de la humedad no es muy notoria. 


\section{Referencias}

1. Wikipedia, Sorghum, http://es.wikipedia.org/wiki/Sorgo.

2. Alberto Chessa, www.ergomix.com.ar., 2007.

3. Elkin Robert G., Freed Marisue B., Hamaker, Bruce R. Condensed Tannins are only partially responsible for variations in nutrient digestibilities of sorghum grain cultivars, Journal of Agricultural and food Chemistry, 1996, 44, 848-853.

4. Roberto Belmar Casso, 1994 www.sian.info.ve/porcinos/publicaciones/encuentros/viii_encuentro/roberto.htm.

5. Claudia Romero, Efecto del pastoreo con bovinos sobre la concentración de taninos condensados en Gliricidia sepium (Jacq) Walp en el trópico seco, 2000, Colima.

6. Maureen Hernández Ángel, Almendro de la India: Potencial biológico valioso, Revista cubana de investigaciones médicas, marzo 2003, 22, 1.

7. Maureen Hernández Ángel, Almendro de la India: Potencial biológico valioso, Revista cubana de investigaciones médicas, marzo 2003, 22, 1.

8. NORMA VENEZOLANA COVENIN3179:1995; Alimentos Para Animales. Determinación De Taninos Condensados.

9. Josephs Wall, Traducido del inglés por Andrés Bottaro; Producción y usos del sorgo.

10. William Benett, Producción moderna sorgo granífero, Noviembre 1991.

11. AGCONNECT, Manual técnico del sorgo, www.viarural.com.ar, marzo 2009

12. W. Russel, Deactivation of tannin in high tannin milo by treatment with urea, Journal Dairy Sci., 1989, $72,2427-2730$

13. Instituto Ecuatoriana de Normalización (INEN); www.inen.gov.ec/normas/norma.php?COD COD_NORMA=518

14. Luis Romero; Silaje de grano húmedo de sorgo: Efecto del contenido de tanino y el tratamiento con urea en la respuesta de vacas lecheras, 2002, EEA INTA. 